\title{
Enantioselective Fluorescence Response of Molecularly Imprinted Polymers (MIPs) toward Carbobenzyloxy- (Cbz) protected Amino Acids
}

\author{
Wei Wan, ${ }^{1}$ Mustafa Biyikal, ${ }^{1}$ Ricarda Wagner, ${ }^{2}$ Börje Sellergren, ${ }^{2}$ and Knut Rurack ${ }^{1}$ \\ ${ }^{1}$ Div. 1.9 Sensor Materials, BAM Federal Institute for Materials Research and Testing, Richard- \\ Willstätter-Str. 11, D-12489 Berlin, Germany, mustafa.biyikal@bam.de \\ ${ }^{2}$ INFU Institute of Environmental Research, Faculty of Chemistry, Technical University of Dortmund, \\ Otto-Hahn-Str. 6, D-44221 Dortmund, Germany
}

\begin{abstract}
Integration of a polymerisable fluorescent urea-nitrobenzoxadiazole monomer 1 into a few-nanometre thin molecularly imprinted polymer (MIP) shell coated onto a $300 \mathrm{~nm}$ silica core with the tetrabutylammonium salt of $\mathrm{N}$-carbobenzyloxy-L-phenylalanine (Cbz- or Z-L-Phe) as the imprinted template yields core/shell MIP nanoparticles that respond to the designated analyte with a strong fluorescence increase in the visible spectral range. The system shows promising imprinting and enantioselectivity factors of 3.6 and 1.9 when benzylmethylacrylate (BMA) is used as co-monomer and ethylene glycol dimethacrylate (EDMA) as cross-linker. A second, naphthalimide-based fluorescent urea 2 is employed in the preparation of MIP thin-films and monolithic polymers for separation techniques. Spectroscopic and chromatographic studies of the MIPs with the analytes Z-L-Phe, Z-Lglutamic acid (Z-L-Glu) and penicilin G (PenG) revealed the (enantio)selective discrimination behaviour of these materials.
\end{abstract}

Key words: enantioselectivity, molecular imprinting, fluorescence, core-shell nanoparticles, chromatography.

Chiral discrimination between enantiomers is receiving constantly growing attention in many analytical applications in prominent fields such as pharmaceutical production, clinical diagnostics and the food sciences. ${ }^{1,2}$ For many chiral biochemically active compounds, one enantiomer exhibits desirable physiological or pharmacological properties, while the other enantiomer may show very different effects. These can range from a different activity in a biochemical reaction or biotechnological process to an actually harmful impact on a living organism. ${ }^{3}$ In particular, in the pharmaceutical industry the search for potent stereo-chemically pure drugs is a strong and market-relevant driver. Many chemical methods to assess the enantiomeric purity of synthetic products have been developed, yet most of them are inherently laboratory-bound and require sampling, transport, clean-up and analysis in a lab environment. ${ }^{1,2}$ Often, a chiral separation element such as a chromatography column fulfils the task required, and molecularly imprinted polymers (MIPs) have become successful tools in this regard. ${ }^{4,5}$ When aiming at rapid screening or analysis in process control environments optical methods-in particular fluorescence-are often the method of choice. ${ }^{6}$

The key step to convert such materials into actual sensor materials would be the implementation of a fluorescent signalling unit into the MIP. Apart from MIPs for fluorescent analytes, ${ }^{7,8}$ approaches to fluorescence displacement assays ${ }^{9,10}$ or the fluorescence tagging of analytes prior to detection in the MIP $^{11,12}$ are not ideal for sensor applications or reduce the possibility for discrimination because of the attachment of an (unspecific fluorophore) tag. In addition, the quenching of dyes-lacking designated receptor sites-covalently embedded into MIPs can only be employed for analytes, which are powerful quenchers. ${ }^{13,14}$ The covalent integration of a fluorescent probe monomer into a MIP however has only seldom 
been accomplished. ${ }^{15-19}$ Especially examples showing directional recognition at a designated binding site or fluorescence enhancement upon analyte binding are scarce. ${ }^{18,19}$ This lack of actual sensing matrices prompted us to develop such probe monomers, ${ }^{20,21}$ and recently we could even report the first fluorogenic MIPs that simply 'light up' upon binding of the analyte in an analytically advantageous wavelength range. $^{20}$ To approximate the aforementioned aim in the context of enantioselective recognition we chose the deprotonated Z-L-Phe as template (Figure 2), a well-known building block in peptide synthesis and frequent target of MIP development. ${ }^{5}$ The impact of D-amino acids on an organism and the importance of their detection are well documented. ${ }^{22,23}$ For the fluorescent monomer 1, we integrated a nitrobenzoxadiazole (NBD) fluorophore carrying a directly fused urea group as the carboxylate recognition site connected through a short ethylene spacer with a polymerisable methacrylate group (Figures 1,2). ${ }^{20}$<smiles>C=C(C)C(=O)CCNC(=O)Nc1ccc([N+](=O)[O-])c2nonc12</smiles>

1

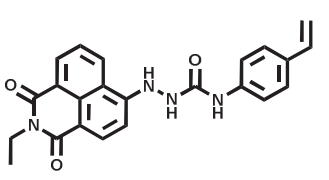

2
Fig. 1. Chemical structures of monomers 1 and 2.

A)
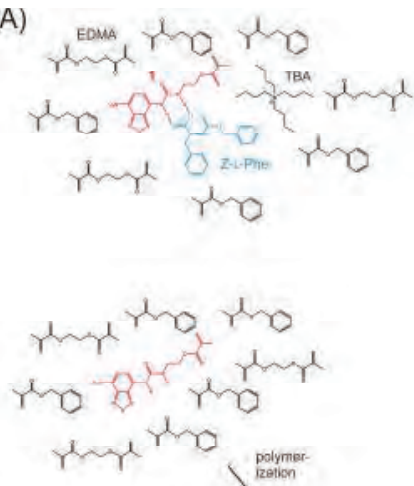

(iii)
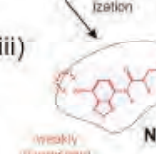

B)

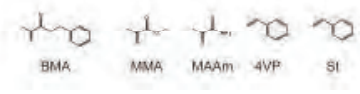

C)

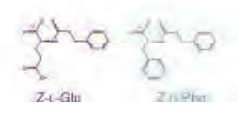

Fig. 2. A) (i) Preparation of the fluorescent MIP with 1. (ii) Switching between high and low fluorescence by extraction and rebinding of the template. (iii) Template-free preparation of the NIP. (iv) Nonspecific binding of the template. (v) Cross-sensitivity of the MIP; B) Co-monomers and C) further analytes investigated in this study.
NBD dyes are known to show intense absorption and fluorescence bands at ca. 450 and $550 \mathrm{~nm}$ when equipped with an electron donating group in the 4-position, induced by an intramolecular charge transfer (ICT) process. $^{24}$ Hence, we introduced the moderately electron donating 4-urea group to bind the electron-rich carboxylate guest by hydrogen-bonding to generate bathochromic shifts and to increase the NBD fluorescence. Monomer 1 was prepared starting from 4-amino-NBD ${ }^{25}$ and methacrylic acid-2-isocyanatoethylester with 4(dimethylamino)-pyridine (DMAP) as catalyst. ${ }^{20}$ A second naphthalimide- ( $\mathrm{NI}$ ) based monomer was prepared starting from 4-bromo-1,8naphthalic anhydride in three steps. ${ }^{21}$ Compared to monomer 1 monomer 2 contains three $\mathrm{NH}$-groups for enhanced hydrogenbonding interactions with carboxylates and emits also in the analytically advantageous region $>500 \mathrm{~nm}$. For molecular imprinting, the selection of a solvent, which is suitable for complex formation between the functional fluorescent monomer and the template molecule in the polymerisation mixture is critical. Investigation of $\mathbf{1}$ with the tetrabutylammonium (TBA) salt of Z-L-Phe in various solvents (acetonitrile, chloroform, tetrahydrofuran) revealed that chloroform is the best choice, because of the most pronounced spectral shifts and fluorescence enhancement induced by complex formation. ${ }^{20}$ Concerning MIP matrix formats, in the case of monomer 1 the best rebinding results were obtained when RAFT (reversible addition-fragmentation chaintransfer) polymerisation of a thin MIP film on silica nanoparticles was employed for sensor layer preparation. ${ }^{20}$ For monomer 2, thin-film MIPs on glass slides, which were activated with 3-methacryloxypropyltrimethoxysilane, proved to be the best format. Here, the polymerisation mixture containing the TBA salt of Z-L-Phe, toluene as solvent, MMA or BMA as comonomer and EDMA as cross-linker, polymerised with the azo-initiator ABDV at high temperatures $\left(>100^{\circ} \mathrm{C}\right)$ in short time, yielded the desired few-micrometre thick films. For comparison purposes, monolithic polymer matrices were prepared of monomer 2 with EDMA as cross-linker. Because of the low solubility of $\mathbf{2}$ in weakly polar organic solvents and its high concentration in the polymerisation mixture, DMF had to be used as solvent for the bulk polymerisation to prevent the formation of aggregated urea probes in the monolithic polymer, which would promote non-specific interactions of the template molecules with the MIP material. After the bulk polymerisation at $45^{\circ} \mathrm{C}$ for $24 \mathrm{~h}$, the template molecules, which were deprotonated with PMP $(1,2,2,6,6$ - 
pentamethylpiperidine), were Soxhlet-extracted from the crushed polymer for $24 \mathrm{~h}$ with $\mathrm{MeOH} / \mathrm{HCl}$.

The challenge of a highly sensitive urea-based MIP is the formation of strong hydrogen bonds between the urea and the carboxylate group and to avoid undesired deprotonation of the urea, which would give rise to non-directional electrostatic interactions at the expense of directional hydrogen-bonding and would thus hamper the formation of cavities containing the urea group of the fluorescent monomer. Deprotonation is observed in titration experiments of ureas and carboxylates by a strongly red-shifted absorption band in the spectra. ${ }^{26}$ In chloroform, monomer 1 showed the development of an absorption band at ca. $520 \mathrm{~nm}$ only after addition of a 7-fold excess of TBA-deprotonated Z-L-Phe, which was tentatively ascribed to deprotonation of the urea group. But before the onset of deprotonation, a ca. $30 \mathrm{~nm}$ bathochromic shift in absorption and a ca. $10 \mathrm{~nm}$ red-shift in fluorescence along with a strong fluorescence enhancement (ca. 100fold) was observed. ${ }^{20}$ The titration data of 1 and Z-L-Phe in chloroform, analysed according to a $1: 1$ binding model, yielded a complex formation constant $\log K=5.11$, which is suitable for imprinting. A similar deprotonation effect was observed in the case of monomer 2 . But compared to 1 more polar solvents like MeCN could be introduced without deprotonation of the monomer 2, signifying that the NBD unit is more electron-accepting than the $\mathrm{NI}$ unit. This allowed also the use of the corresponding MIPs in chromatographic applications in polar solvents. According to the titration experiments the complex formation constant of $2 / Z-L-$ $\mathrm{Phe} / \mathrm{PMP}$ in $\mathrm{MeCN}$ was $\log K=3.08 .^{21}$

Core/shell nanoparticles were selected for monomer 1 as the format because these substrates are a very versatile platform to be implemented with many layered as well as fluidic setups. Further benefits of the thin polymer shells on robust core particles are the reproducible surface-initiated polymerisation, ${ }^{27}$ their fast response times and permission to extract the template quantitatively from the cavities in comparatively short time. ${ }^{28}$ This strategy has two basic advantages, namely a higher density of the silica core, which allows facile handling of the beads in many different applications and the fact that silica nanoparticles are well-suited for use with optical detection techniques. Besides 1 and Z-L-Phe, EDMA was used as a moderately polar crosslinker and various co-monomers of different polarity were employed (Fig. 2). To form a homogeneous polymer layer around the $\mathrm{SiO}_{2}$ nanoparticles, 2-(2-cyanopropyl)dithiobenzoate (CPDB) as RAFT agent was coupled onto the amino-silane-activated $\mathrm{SiO}_{2}$ surface. Figure 3 shows a representative TEM image of the highly mono-disperse core/shell MIP particles CS1, revealing a shell thickness of $10 \mathrm{~nm}$.

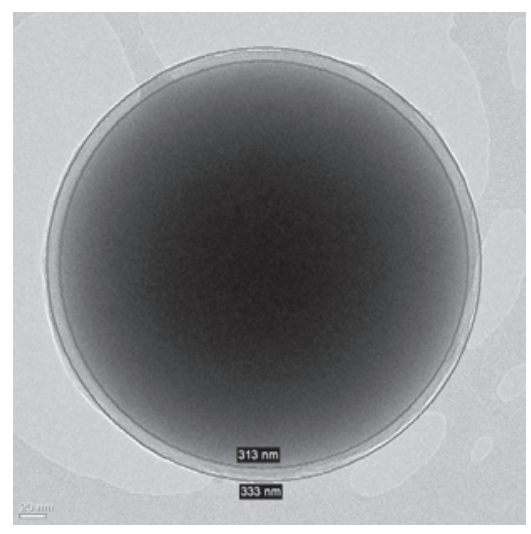

Fig. 3. TEM image of CS1

The (enantio)selectivity of the nanoparticle sensors was tested in chloroform by titrating the MIP particles with the TBA salt of Z-L-Phe, the corresponding $\mathrm{D}$-isomer as the enantiomeric twin and the conformationally closely designed amino acid Z-L-Glu containing two possible hydrogen-bonding sites. According to the results in Table 1, the MIPs prepared with benzylmethylacrylate (BMA) as the comonomer yielded the highest imprinting factor and the best (enantio)selectivity.

Tab. 1: 1:Z-L-Phe ratio (1:1), cross-linker = EDMA, porogen = chloroform Cr./Co. = cross-linker $/$ comonomer; a MIP/NIP discrimination factor, ${ }^{b}$ enantioselectivity factor, ${ }^{c} \boldsymbol{1}$ was diluted 5-fold, ${ }^{d}$ porogen $=$ chloroform $/$ heptane $(7: 3),{ }^{e} 1: 1$ ratio .

\begin{tabular}{|c|c|c|c|c|c|}
\hline No. & $\begin{array}{c}\text { Co- } \\
\text { mon. }\end{array}$ & $\begin{array}{c}\text { Cr./Co. } \\
\text { ratio }\end{array}$ & $\begin{array}{c}\text { Flu. } \\
\text { Enh. }\end{array}$ & MIP/NIP $^{\mathrm{a}}$ & L/D $^{\mathrm{b}}$ \\
\hline CS1 & BMA & $5: 1$ & 2.5 & 3.6 & 1.90 \\
\hline CS2 & MMA & $5: 1$ & 1.8 & 2.8 & 1.20 \\
\hline CS3 & VP & $4: 1$ & 1.04 & 1 & - \\
\hline CS4 $^{\mathrm{c}}$ & MMA & $5: 1$ & 1.2 & 1 & - \\
\hline CS5 & Sty:MMA $^{\mathrm{e}}$ & $5: 1$ & 1.8 & 2.2 & 1.50 \\
\hline CS6 & MAAm & $5: 1$ & - & - & - \\
\hline CS7 $^{d}$ & BMA & $5: 1$ & 1.7 & 3.1 & 1.20 \\
\hline CS8 $^{d}$ & BMA & $5: 1$ & 1.4 & 2.4 & 1.32 \\
\hline
\end{tabular}


The beneficial effect is ascribed to the presence of the benzyl groups, offering additional aromatic moieties for further $\pi-\pi$ interactions with the phenyl groups of Phe and the Zprotecting group in the cavities.

In Figure 4, the different responses of CS1-MIP and NIP and the discrimination against the two other protected amino acids are illustrated. The maximum fluorescence enhancement that could be obtained for CS1 and Z-L-Phe amounted to a factor of ca. 2.5. This value is considerably smaller than the value of ca. 100 found at an optimum ratio of $1: Z$-L-Phe $=1: 5$ in the molecular solution studies and is tentatively ascribed to the following reasons.

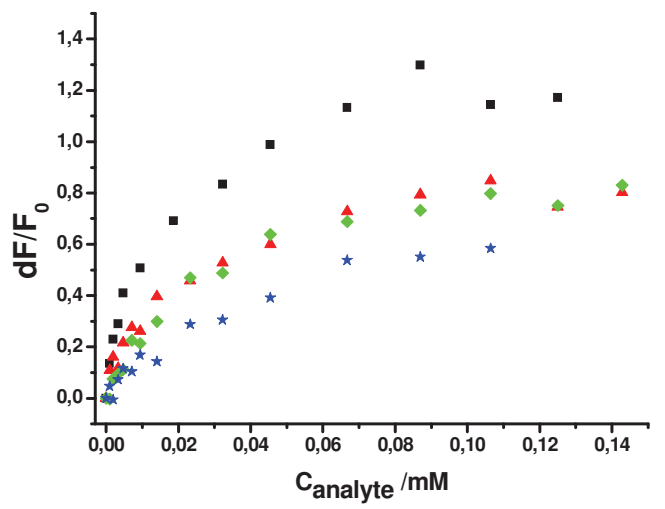

Fig. 4. Normalized fluorescence changes $d F / F_{0}$ (with $\left.d F=F-F_{0}\right)$ vs. concentration for CS1 and Z-L-Phe (black squares), CS1 and Z-D-Phe (green diamonds), CS1 and Z-L-Glu (red triangles) and CSN1 and Z-L-Phe (blue stars); CSN1 are the NIP analoga of CS1.

On one hand, the absorption maximum was found at $412 \mathrm{~nm}$ for 1 in CS1 suspended in chloroform, which is distinctly red-shifted compared with 1 in chloroform (403 nm) or toluene (404 $\mathrm{nm}$ ) and lies closer to the maxima of 1 in THF (413 nm), MeCN and methanol (both $411 \mathrm{~nm}$ ). If we further consider that the fluorescence quantum yield $\Phi_{f}$ of NBD dyes increases with increasing solvent polarity ${ }^{24}$ and $\Phi_{\mathrm{f}}(\mathbf{1})$ was determined here to 0.002 in chloroform, 0.01 in THF and 0.024 in MeCN, a $\Phi_{\mathrm{f}}=0.015$ for $\mathrm{CS1}$ seems to underline the conclusion drawn from the absorption measurements. On the other hand, the maximum of the fluorescence of CS1, occurring at $496 \mathrm{~nm}$ compared with $493 \mathrm{~nm}$ for 1 in chloroform and $502 \mathrm{~nm}$ for 1 in MeCN, suggests that confinement of $\mathbf{1}$ in the polymer matrix does not allow for full relaxation of the excited dye. These three observations point to the conclusion that confinement of the dye in the polymer matrix entails an intrinsically higher fluorescence in the absence of the analyte and explains the lower enhancement observed. Note that template extraction from the thin shell as monitored by UV/vis measurements at 265 $\mathrm{nm}$ (absorption band of Z-L-Phe) was virtually quantitative. Analytical studies of CS1 finally revealed a limit of detection in a cuvette-based experiment of ca. $1 \mu \mathrm{M}$ for Z-L-Phe in chloroform.

Although the MIP particles showed high (enantio)selectivity in organic solvents, binding studies in water did not yield a measureable response. It is assumed that the thin polymer shell of the nanoparticles is not hydrophobic enough to shield the fluorescent urea 1 against competing water molecules. Thus, a two phase system was developed, consisting of chloroform and water, to detect Z-L-Phe indirectly in water. CS1 (10 mg) was suspended in a $10 \mathrm{~mm}$ quartz cell in chloroform $(2 \mathrm{~mL})$. Subsequently, water (1 $\mathrm{mL}$ ) was added to form the two phase system. Under stirring, $0.1-1.4 \mathrm{mmol}$ of Z-L-Phe were then added, and the two phases were left to settle for $5 \mathrm{~min}$ before measuring the fluorescence. As a control experiment the amount of Z-L-Phe that remained in the water phase was measured by HPLC. Despite the presence of water as the second phase, the fluorescence "lighting-up" response, the (enantio)selectivity and the fast response time of CS1 with Z-L-Phe was preserved.

In contrast to monomer $\mathbf{1}$, monomer $\mathbf{2}$ shows a decrease of the emission through deprotonation, anion binding or by increasing the polarity of the solvent. Thus, for the preparation of the monolithic polymers, three weakly basic molecules were chosen to serve as templates in the bulk polymerisation. Z-L-Glu and Z-L-Phe were deprotonated with PMP and PenG was used as its procain salt. After the bulk polymerisation, materials with distinct colour differences were obtained (Fig. 5). The much darker colours like in the case of $\mathbf{M}_{\mathbf{P h e}}$ and $\mathbf{M}_{\mathrm{Glu}}$ indicate the higher degree of deprotonation of the ureas in the polymer.

After the extraction, orange materials remained, which indicated the successful removal of templates from the polymer matrices. The crushed materials (particle sizes of 25-50 $\mu \mathrm{m}$ ) were investigated by HPLC techniques. According to the chromatography results, $\mathbf{M}_{\mathrm{Glu}}$ was able to discriminate between the enantiomers with a retention time of $5.38 \mathrm{~min}$ for L- and 4.37 min for the D-enantiomer of ZGlu in MeCN/water (1:1). In pure MeCN, the MIP showed a chromatographic imprinting factor of $\mathrm{IF}_{\mathrm{C}}=3.32$. In contrast to $\mathbf{M}_{\mathrm{Glu}}, \mathbf{M}_{\mathrm{Phe}}$ showed no enantioselective discrimination in 
water-containing MeCN. But in pure $\mathrm{MeCN}$, the retention time of Z-L-Phe was significantly higher than for the D-enantiomer $\left(\mathrm{IF}_{\mathrm{C}}=9.85\right)$. Also in the case of $\mathbf{M}_{\text {Pen }}$ a strong imprinting effect was observed in MeCN/water mixtures.

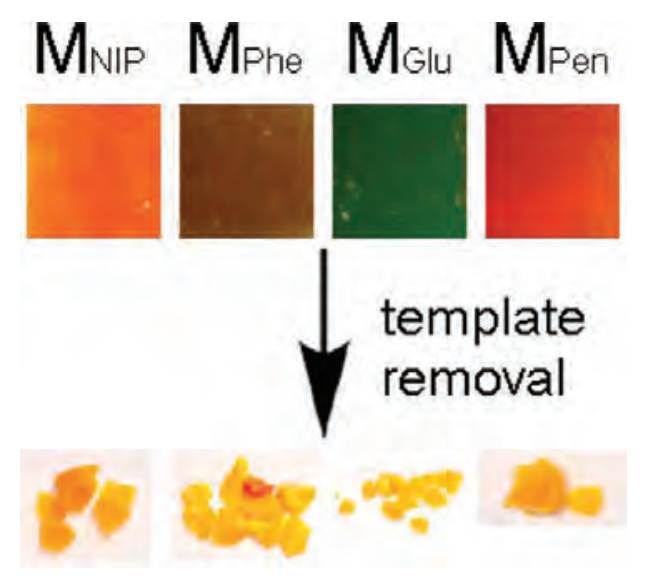

Fig. 5. Colours of monolithic polymers synthesized from 2 in the presence of Z-L-Phe, Z-L-Glu, and PenG prior and after Soxhlet extraction.

For the preparation of the thin-film MIPs, the TBA salt of Z-L-Phe was introduced to increase the nucleophilic character of the carboxylate group to improve the hydrogen-bonding to the urea group. The rebinding experiments were conducted in MeCN and, according to the results of the fluorescence titrations, an imprinting factor of $\mathrm{IF}_{\mathrm{c}}=3.4$ and $\mathrm{a}$

\section{References}

[1] N. M. Maier, P. Franco, W. Lindner, J. Chromatogr. A 906, 3-33 (2001).

[2] E. L. Izake, J. Pharm. Sci. 96, 1659-1676 (2007).

[3] S. W. Smith, Toxicol. Sci. 110, 4-30 (2009).

[4] N. M. Maier, W. Lindner, Anal. Bioanal. Chem. 389, 377-397 (2007).

[5] J. Z. Hilt, M. E. Byrne, Adv. Drug Deliv. Rev. 56, 1599-1620 (2004).

[6] D. Leung, S. O. Kang, E. V. Anslyn, Chem. Soc. Rev. 41, 448-479 (2012).

[7] A. M. Rosengren, B. C. G. Karlsson, I. Naslund, P. O. Andersson, I. A. Nicholls, Biochem. Biophys. Res. Commun. 407, 60-62 (2011).

[8] F. J. Sainz-Gonzalo, A. L. Medina-Castillo, J. F. Fernandez-Sanchez, A. Fernandez-Gutierrez, Biosens. Bioelectron. 26, 3331-3338 (2011). discrimination factor of $\mathrm{q}_{\mathrm{Phe}}=1.7$ of the L/Denantiomers was determined at $0.5 \mathrm{mM}$ template concentration.

Our recent works have demonstrated for the first time that fluorogenic monomers can be integrated into silica core/MIP shell nanoparticles and thin MIP films on glass supports, absorbing and fluorescing in an analytically important wavelength range. These sensor matrices can be designed so that a specific fluorescence decrease or a fluorescence 'light up' signal is obtained upon binding to the analyte. The amount of nonspecific binding and the observed enantio(selectivity) are promising when compared with previous values of $<2$ that have been published in the literature for MIP sensing systems utilizing fluorescence. ${ }^{17,29}$ Handling of the sensory particles and the film-covered strips is straightforward and the response times in the assay are fast, in particular in the particle case. Relevant sensing scenarios can be approximated with an extraction-based, biphasic protocol. We have also demonstrated that the thin-film- and monolithic MIPs are able to discriminate and separate enantiomers in mixed aqueous organic solvents and that such a system can be directly employed in pharmaceutical process-control, which allows the detection of analytes in aqueous solution and broadens the application to organic acids more relevant in environmental analysis.

[9] K. Haupt, A. G. Mayes, K. Mosbach, Anal. Chem. 70, 3936-3939 (1998).

[10] J. L. Urraca, M. C. Moreno-Bondi, G. Orellana, B. Sellergren, A. J. Hall, Anal. Chem. 79, 4915-4923 (2007).

[11] D. Kriz, O. Ramstrom, A. Svensson, K. Mosbach, Anal. Chem. 67, 2142-2144 (1995).

[12] C. F. Chow, M. H. W. Lam, M. K. P. Leung, Anal. Chim. Acta 466, 17-30 (2002).

[13] Y. Liao, W. Wang, B. H. Wang, Bioorg. Chem. 27, 463-476 (1999).

[14] R. Y. Liu, G. J. Guan, S. H. Wang, Z. P. Zhang, Analyst 136, 184-190 (2011).

[15] J. Li, C. E. Kendig, E. E. Nesterov, J. Am. Chem. Soc. 129, 15911-15918 (2007).

[16] G. Gupta, A. S. B. Bhaskar, B. K. Tripathi, P. Pandey, M. Boopathi, P. V. L. Rao, B. Singh, R. Vijayaraghavan, Biosens. Bioelectron. 26, 25342540 (2011).

[17] P. Turkewitsch, B. Wandelt, G. D. Darling, W. S. Powell, Anal. Chem. 70, 2025-2030 (1998). 
[18] T. H. Nguyen, R. J. Ansell, Org. Biomol. Chem. 7, 1211-1220 (2009).

[19] H. Kubo, N. Yoshioka, T. Takeuchi, Org. Lett. 7, 359-362 (2005).

[20] W. Wan, M. Biyikal, R. Wagner, B. Sellergren, K. Rurack, Angew. Chem. Int. Ed. 52, 7023-7027 (2013).

[21] R. Wagner, W. Wan, M. Biyikal, E. Benito-Peña, M. C. Moreno-Bondi, I. Lazraq, K. Rurack, B. Sellergren, J. Org. Chem. 78, 1377-1389 (2013).

[22] K. Hamase, A. Morikawa, K. Zaitsu, J. Chromatogr. B 781, 73-91 (2002).

[23] K. Hamase, A. Morikawa, S. Etoh, Y. Tojo, Y. Miyoshi, K. Zaitsu, Anal. Sci. 25, 961-968 (2009).

[24] S. Fery-Forgues, J.-P. Fayet, A. Lopez, J. Photochem. Photobiol. A Chem. 70, 229-243 (1993).

[25] S. Uchiyama, T. Santa, T. Fukushima, H. Homma, K. Imai, J. Chem. Soc., Perkin Trans. 2 2165-2173 (1998).

[26] A. B. Descalzo, K. Rurack, H. Weisshoff, R. Martínez-Máñez, M. D. Marcos, P. Amorós, K. Hoffmann, J. Soto, J. Am. Chem. Soc. 127, 184200 (2005).

[27] R. Barbey, L. Lavanant, D. Paripovic, N. Schüwer, C. Sugnaux, S. Tugulu, H.-A. Klok, Chem. Rev. 109, 5437-5527 (2009).

[28] S. Tokonami, H. Shiigi, T. Nagaoka, Anal. Chim. Acta 641, 7-13 (2009).

[29] N. Yoshioka, T. Takeuchi, Org. Lett. 7, 359-362 (2005). 